\title{
Über Permolybdate.
}

\author{
von \\ W. Muthmann und W. Naget.
}

Schon vor längerer Zeit ist die Beobachtung publiziert worden, dafs Molybdänsäure und saure Molybdate mit Wasserstoffsuperoxyd tief gelb gefärbte Lösungen geben, ${ }^{1}$ und es hat BäRWALD ${ }^{2}$ zuerst versucht, die dabei entstehenden Körper krystallisiert zu erhalten. Er verfuhr zunächst in der Weise, dafs er gewöhnliches Ammonmolybdat, sowie das entsprechende Kaliumsalz in Wasserstoffsuperoxyd löste, eindunsten liefs und die erhaltenen Krystalle analysierte. Die von ihm aufgestellten Formeln waren aber so komplizierte, dafs seine Angaben kaum beachtet wurden; für das Ammonium- und Kaliumsalz fand er $18 \mathrm{MoO}_{3} \cdot 14 \mathrm{NH}_{3} \cdot 3 \mathrm{H}_{2} \mathrm{O}_{2} \cdot 18 \mathrm{H}_{2} \mathrm{O}$ resp. $16 \mathrm{MoO}_{3} \cdot 6 \mathrm{~K}_{2} \mathrm{O}$. $4 \mathrm{H}_{2} \mathrm{O}_{2} \cdot 13 \mathrm{H}_{2} \mathrm{O}$. Wie man sieht, nimmt BäRWaLD an, dafs das Wasserstoffsuperoxyd als solches, ähnlich wie Krystallwasser, in seinen Salzen vorhanden sei.

Die Versuche von BärwaLd wurden später in genau analoger Weise von Péchard ${ }^{3}$ wiederholt, und zwar mit dem Erfolge, dals er zu äufserst einfachen Formeln für die in Frage stehenden Körper gelangte. Nach seinen Publikationen liegen nämlich Analoga der Persulfate vor, und er giebt für beide Salze die Formeln $\mathrm{KMoO}_{4} \cdot 2 \mathrm{H}_{2} \mathrm{O}$ resp. $\mathrm{NH}_{4} \mathrm{MoO}_{4} \cdot 2 \mathrm{H}_{2} \mathrm{O}$ an. Später wurden die beiden Salze nochmals dargestellt, und zwar von MöLLER; ${ }^{4}$ derselbe hat, allerdings nach einer veralteten und längst als unbrauchbar erkannten Methode, ${ }^{5}$

1 Werther, Journ. pr. Chem. (1861) 83, 198 und Sснӧns, Zeitsehr. anal. Chem. (1870) 9, 41 und 311.

2 Dessen Inaugural-Dissertation: „Beiträge zur Kenntnis des Molybdäns“ (Berlin 1885). Ref. Chem. Centralbl. 1885, 424.

${ }^{3}$ Compt. rend. 112, 720 und Ann. Chim. Phys. (1893) 28, 537.

4 Zeitschr. phys. Chem. (1893) 12, 555.

${ }^{5}$ Reduktion der Säure zu Dioxyd. 
Molybdänbestimmungen ausgeführt, PénARD's Formeln bestätigt und zugleich mit Hilfe von Leitfähigkeitsmessungen nach WALDEN's Valenzregel bestimmt, dafs die Übermolybdänsäure analog der Überschwefelsäure zweibasisch ist, also die Formel $\mathrm{H}_{2} \mathrm{Mo}_{2} \mathrm{O}_{8}$ besitzt. Damit schien alles in der schönsten Ordnung, umsomehr als Péchand in der zweiten der oben citierten Abhandlungen eine ganze Reihe von vorzüglich stimmenden Analysen angiebt, und es sind infolgedessen die Angaben der beiden letztgenannten Chemiker in die meisten neueren Lehrbücher aufgenommen worden. ${ }^{1}$

Beim Durchlesen der citierten Arbeiten fällt zunächst auf, dafs die krystallographischen Angaben einigermafsen von einander abweichen. Péchard hat sein Ammoniumsalz von Dufer ${ }^{2}$ messen lassen; dieser überaus zuverlässige und geübte Krystallograph fand folgende Konstanten:

$$
\text { Monoklin, } a: b: c=1.46822: 1: 1.02585 \beta=105^{0} 45^{\prime} 40^{\prime \prime} \text {. }
$$

Als Formel ist $\mathrm{NH}_{4} \mathrm{MoO}_{4} \cdot 2 \mathrm{H}_{2} \mathrm{O}$ angegeben.

Das von MöLLer erhaltene Salz hat Fock ${ }^{3}$ untersucht; obwohl nun jener genau nach PECHARD's Angaben arbeitete, fand dieser ganz andere Zahlen als Dufet, nämlich:

Monoklin, $a: b: c=0.4693: 1: 0.2956 \beta=112^{\circ} 39^{\prime} 30^{\prime \prime}$.

Allerdings ist in der Fock'schen Arbeit als Formel $\mathrm{NH}_{4} \mathrm{MoO}_{4}$, also wasserfreies Salz, angegeben, doch dürfte dies wohl auf einen Schreibfehler zurückzuführen sein.

Nun existiert aber noch eine dritte Messung des Ammoniumpermolybdates, nämlich diejenige BärwaLd's. Trotzdem dessen Formel von der Pechard's völlig verschieden ist, findet er genau dieselben krystallographischen Konstanten, wie DDFET, nämlich:

$$
\text { Monoklin, } a: b: c=1.4727: 1: 1.0268 \beta=105^{\circ} 28^{\prime} \text {. }
$$

Die geringen Differenzen sind selbstredend auf Beobachtungsfehler zurückzuführen. Es leuchtet also ein, dal's PÉCHARD und BäRwaLd denselben Körper unter den Händen gehabt haben, obwohl ihre Analysen aufserordentlich differieren:

1 Riouten's Lehrbuch der anorg. Chemie. (8. Aufl.) S. 456.

2 Zeitschr. Krystallogr. (1894) 22, 594.

${ }^{3}$ Ebendaselbst 22, 32. 


\begin{tabular}{|c|c|c|c|}
\hline \multicolumn{3}{|c|}{ Pechard: } & BÄRWALD: \\
\hline $\mathrm{MoO}_{3}$ & 67.31 & $67.27^{\circ} \%$ & $79.46 \quad 79.44 \%$ \\
\hline$\left(\mathrm{NH}_{4}\right)_{2} \mathrm{O}$ & 12.11 & $12.18 n$ & $11.18 \%$ \\
\hline 0 & 3.74 & 3.74 & 1.47, \\
\hline
\end{tabular}

und es folgt daraus ohne weiteres, dals wenigstens eine der Angaben unrichtig sein muls.

Auf der anderen Seite findet man da, wo nach den Analysenresultaten Identität der Körper angenommen werden sollte, gänzlich sich widersprechende krystallographische Angaben, und es schien daher nötig, die Sache einer crneuten Untersuchung zu unterwerfen. Das Resultat war ein sehr merkwürdiges; dals nämlich von allen bis jetzt publizierten Analysen übermolybdünsaurer Salze nicht eine einzige richtig ist. Es sei uns daher gestattet, zunächst die von uns angewendeten analytischen Methoden zu beschreiben.

Das Molybdän wägt man am besten als Trioxyd. Wenn Ammonsalze vorliegen, genügt einfaches Erhitzen mit dem Bunsenbrenner, wobei reines $\mathrm{MoO}_{3}$ zurückbleibt; die Temperatur darf natürlich Rotglut nicht erreichen. Bei den Kaliumsalzen haben wir das Molybdän als $\mathrm{MoS}_{3}$ gefällt und dieses durch längeres, vorsichtiges Rösten in $\mathrm{MoO}_{3}$ übergeführt, eine Hethode, welche von FrIfDhEIM herrührt und äul'serst genaue Resultate liefert.

Pechard trennte die Molybdänsäure von den Alkalien durch Erhitzen der trockenen Substanz im Salzsäurestrom auf $300-500^{\circ}$; alles Molybdün verflüchtigt sich als $\mathrm{MoO}_{3} \cdot 2 \mathrm{HCl}$ und die Chloride der Alkalien bleiben zurück. Von der Anwendbarkeit dieser Hethode haben wir uns überzeugt und gute Resultate erhalten; sie wurde in einzelnen Fällen zur Bestimmung der fixen Alkalien benutzt.

Der Sauerstoff läfst sich, wie schon BärwaLd fand, ganz bequem durch Titrieren mit Permanganat in schwefelsaurer Lösung bestimmen; die Permolybdänsäure reagiert nämlich mit Übermangansüure genau wie Wasserstoffsuperoxyd. Es wird die Chamäleonlösung unter lebhafter Sauerstoffentwickelung entfärbt, und die Endreaktion ist ganz scharf. PrCHard bestimmte den Sauerstoff volumetrisch nach Erbitzen der Substanz im Vakuun; diese unnötig komplizierte Methode haben wir nicht probiert, wohl aber uns von der Richtigkeit der Permanganatmethode überzeugt, indem wir die Analysen mehrfach jodometrisch kontrollierten: das mit Salzsäure entwickelte Chlor wurde in Jodkaliumlösung geleitet, und das Jod titriert. Die Übereinstimmung war völlig befriedigend. 
Ammoniak wurde destilliert und titriert, Kalium entweder als $\mathrm{KCl}$ oder $\mathrm{K}_{2} \mathrm{SO}_{4}$ gewogen.

\section{Ammoniumsalze.}

Zur Darstellung derselben löst man man gewöhnliches Ammonmolybdat in $20 \%$ igem Wasserstoffsuperoxyd auf. Letzteres muls sorgfältig gereinigt sein; alle von uns versuchten, käuflichen Präparate enthielten so viel Kieselsäure und Thonerde, dafs Destillation und Ausäthern nötig war.

Trägt man das Salz $\left(\mathrm{NH}_{4}\right)_{8} \mathrm{Mo}_{7} \mathrm{O}_{24} \cdot 4 \mathrm{H}_{2} \mathrm{O}$ in der Kälte bis zur Sättigung ein, so erhält man eine sehr intensiv gelbrot gefärbte Flüssigkeit, die beim Stehen über Phosphorpentoxyd sicher nach kurzer Zeit zu krystallisieren beginnt. Das ausgeschiedene Produkt besteht zunächst aus orangerot gefärbten, schönen monoklinen Krystallen; die Farbe dieser ersten Fraktion ist nicht citronengelb, wie die meisten Beobachter angeben. Erst nach einiger Zeit entstehen Krystalle von dieser Farbe, und wenn man eine nach obiger Vorschrift bereitete Lösung längere Zeit stehen lälst, so resultiert ein Krystallgemisch zweier, sowohl durch Farbe, als auch durch Krystallform deutlich verschiedener Substanzen. Einige Versuche lehrten uns bald die Bedingungen kennen, unter denen die Körper einheitlich erhalten werden können: Aus konz. Wasserstoffsuperoxydlösungen erhält man die roten, aus verdünnteren dagegen die citronengelben Krystalle.

Der orangerote Körper besitzt die Formel

$$
3\left(\mathrm{NH}_{4}\right)_{2} 0.7 \mathrm{MoO}_{4}+12 \mathrm{H}_{2} \mathrm{O} \text {. }
$$

Die zur Analyse benutzte Portion war erhalten worden durch zweitägiges Stehen einer gesättigten Lösung in 20\% igem Wasserstoffsuperoxyd über Phosphorpentoxyd. Die Krystalle wurden genau auf Einheitlichkeit unter dem Mikroskop geprüft. Sie wurden von der Mutterlauge durch Abspülen mit Äther getrennt und zwischen Fliefspapier getrocknet.

Eine Portion war aus wenig Wasser umkrystallisiert worden.

Die Analyse ergab folgendes:

$$
\begin{aligned}
& \text { Berechnet: }
\end{aligned}
$$

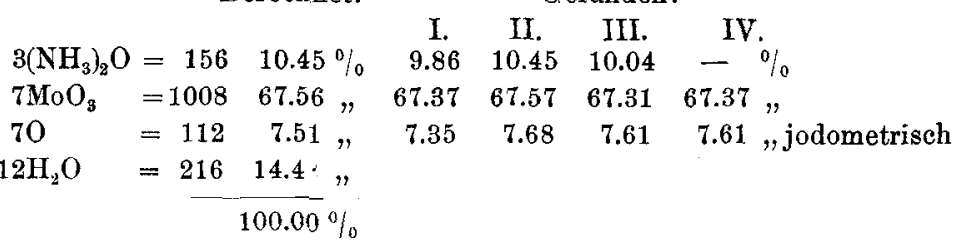


Die obigen Analysen beziehen sich auf drei verschiedene Portionen; I. war aus einem Gemisch der rotgelben und citronengelben Krystalle ausgelesen, II. und III. direkt aus der sehr konz. Lösung auskrystallisierte reine Produkte, und IV. endlich aus kaltem Wasser umkrystallisiert.

Die citronengelben Krystalle ergaben bei der Analyse die

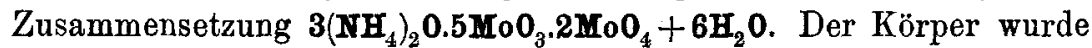
dargestellt durch Eindunsten der Mutterlauge von den orangeroten Krystallen, und ebenfalls teilweise aus möglichst wenig kaltem Wasser umkrystallisiert.

Die Analyse ergab:

\begin{tabular}{lrrrrrrr} 
& Berechnet: & \multicolumn{5}{c}{ Gefunden: } \\
& & I. & II. & III. & IV. & \\
$\left(\mathrm{NH}_{4}\right)_{2} \mathrm{O}$ & $11.97 \%$ & 12.22 & 11.38 & 11.72 & - & $\%$ \\
$\mathrm{MoO}_{8}$ & 77.30, & 77.12 & 77.27 & 77.13 & & - & $"$ \\
$\mathrm{O}$ & 2.45, & 2.34 & 2.14 & 2.58 & 2.19 & 2.34 & $2.31 "$ \\
$\mathrm{H}_{2} \mathrm{O}$ & 8.28, & & & & & &
\end{tabular}

Kaliumpermolybdat, $\mathrm{K}_{2} 0.2 \mathrm{MoO}_{3} \cdot \mathrm{MoO}_{4}+3 \mathrm{H}_{2} \mathrm{O}$.

Behufs Darstellung von Kaliumverbindungen gingen wir zunächst aus von dem $7 / 3$-fach sauren Salz $3 \mathrm{~K}_{2} \mathrm{O}_{1} .7 \mathrm{MoO}_{3}$, das in seiner Zusammensetzung dem Ammoniumsalz entspricht und durch $\mathrm{Zu}-$ sammenschmelzen entsprechender Mengen Kaliumkarbonat und Molybdänsäure gewonnen wurde. Die Schmelze wurde gepulvert und in $20 \%$ igem Wasserstoffsuperoxyd gelöst; es /gelang jedoch nicht, aus der tief gefärbten Flüssigkeit Krystalle zu erhalten. Beim Eindunsten im Vakuum schied sich immer ein amorphes, orangefarbenes Pulver ob, das sehr sauerstoffreich war, aber einen konstanten Gehalt an Kalium und Molybdänsäure nicht zeigte.

Wir haben sodann das Kaliumtrimolybdat als Ausgangsmaterial gewählt, weil dies als das beständigste aller Kaliummolybdate am meisten Aussicht auf Erfolg versprach. $10 \mathrm{~g}$ Kaliumkarbonat wurden mit $20 \mathrm{~g}$ reinster, ammoniakfreier Molybdänsäure zusammengeschmolzen, die gepulverte Schmelze mit kochendem Wasser extrahiert und der bald entstehende Krystallbrei von $\mathrm{K}_{2} \mathrm{Mo}_{3} \mathrm{O}_{10} \cdot 3 \mathrm{H}_{2} \mathrm{O}$ abgesaugt und ausgewaschen.

Aus der Lösung dieses Körpers in $20 \%$ igem Wasserstoffsuperoxyd schied sich nur anfänglich in ganz geringen Mengen der amorphe Körper aus; das Filtrat von diesem Niederschlage wurde über Phosphorpentoxyd eingeengt, und in die konz. Lösung eine Spur 
des Ammoniumsalzes eingeimpft, weil wir auf diese Weise den Beginn einer Krystallisation einzuleiten hofften. In der That bedeckte sich nach einiger Zeit der Boden des Gefälses mit schönen, sehr kleinen, stark glänzenden Krystallen, die dem monoklinen System angehörten und eine hellgelbe Farbe zeigten, ganz ähnlich dem oben beschriebenen, sauerstoffarmen Ammoniumsal\%. Die Zusammensetzung war jedoch eine andere als bei jenem.

Die Anityse ergab $\mathrm{K}_{2} \mathrm{O} .2 \mathrm{NoO}_{3} \mathrm{MuO}_{4}+3 \mathrm{H}_{2} \mathrm{O}$.

Berechnet:

$$
\begin{aligned}
& \text { Gefunden: } \\
& \text { I. II. } \\
& \mathrm{K}_{2} \mathrm{O}=9 \pm 15.75 \% \quad 15.35 * \quad 16.17 * * \%
\end{aligned}
$$

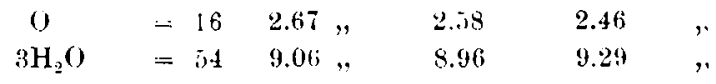$$
3 \mathrm{HoO}_{3}=439 \text { 2 } 72.48, \quad 71.98 \quad 72.19 \quad 72.13 \quad,
$$$$
99.98 \%
$$

* Als $\mathrm{K}_{\mathrm{S}} \mathrm{SO}_{4}$. ** Als $\mathrm{KCl}$ nach Péchand's Methode.

Bei den drei beschriebenen Salzen fällt zunächst auf, dafs in denselben das Verhältnis von Sauerstoff zu Molybdän durchaus kein konstantes ist, wie es doch sein mülste, wemn eine Übermolybdänsäure von einheitlicher Zusammensetzung existierte. Es beträgt dies Verhältnis nämlich in

$$
\begin{aligned}
& \mathrm{NH}_{4}-\mathrm{Salz} \mathrm{I} \quad 1: 1 \text {. } \\
& \begin{array}{cr}
\text { K-Salz } & \text { II } 2: 7 . \\
& 1: 3 .
\end{array}
\end{aligned}
$$

Wäre Pechard's angebliche Permolybdänsäure in den Salzen enthalten, so mürste das Sauerstoffverhältnis stets $1: 2$ sein, was aber in keinem Falle zutrifft. Es fragt sich nun, welche Konstitution den beschriebenen Körpern zukommt.

Zunächst ncigten wir z’ar Anschauung BärwaLD's, dafs in den liörpern einfach Wasserstoffsuperoxyd als solches enthalten sei, und zwar in einer Form wie Krystallwasser in Salzen. Es spricht vieles zu Gunsten einer solchen Annahme; vor allen Dingen zeigen die Salze in wässeriger Lösung sämtliche Reaktionen des Wasserstoffsuperoxyds, allerdings in mebr oder weniger abgeschwächtem Marse. Wir haben beispielsweise folgendes beobachtet: worden.

1. Die Reaktion mit $\mathrm{KMnO}_{4}$ ist bereits oben beschrieben

2. Mit Chromsäure in schwefelsaurer Lösung entsteht die bekannte Blaufärbung durch Überchromsäure, durch Äther extrahier- 
bar; dann sehr bald Reduktion zu Chromisalz. Am besten gelingt der Versuch beim Erwärmen der Lösungen.

3. Die bekannte Reaktion auf $\mathrm{H}_{2} \mathrm{O}_{2}$ mit einer sehr verdünnten Lösung von Kaliumdichromat, Anilin und Oxalsäure (rotviolette Färbung) geben auch die beschriebenen Salze, allerdings erst beim Erwärmen.

4. Aufserordentlich empfindlich sind die Salze gegen Alkalien. Man braucht nur einen Tropfen Kalilauge, Ammoniak oder dergleichen zuzufügen, um sofortige Entfärbung unter lebhafter Sauerstoffentwickelung herbeizuführen. Auch dieses Verhalten erinnert an das Wasserstoffsuperoxyd.

Gegen die Annahme von $\mathrm{H}_{2} \mathrm{O}_{2}$ in Krystallwasserform dagegen spricht die gelbe Farbe der Lösungen, welche beweist, daf's auch in Lösung der Sauerstoff an Molybdänsäure gebunden ist; den Beweis dafür lieferte folgender von uns angestellter Versuch:

Eine feingepulverte Portion des Salzes $3\left(\mathrm{NH}_{4}\right)_{2} \mathrm{O} .7 \mathrm{MoO}_{4} \cdot 12 \mathrm{H}_{2} \mathrm{O}$ wurde auf Thon über Phosphorpentoxyd im Exsiccator im Vakuum drei Wochen lang aufbewahrt und nach Ablauf dieser Zeit analysiert. Es stellte sich heraus, dal's vor allem das Verhältnis von $\mathrm{O}:$ Mo konstant geblieben war; der Körper enthielt $8.54 \% \mathrm{O}$ und $75.45 \% \mathrm{MoO}_{3}$. Dagegen war Ammoniak und Wasser fortgegangen; der $\mathrm{H}_{2} \mathrm{O}$-Gehalt war zurückgegangen auf $4.68 \%$, was nicht hinreicht, um das Vorhandensein des Sauerstoffes in Form von $\mathrm{H}_{2} \mathrm{O}_{2}$ zu erklären.

Die einzige Annahme, welche die Zusammensetzung und die Reaktionen in genügender Weise erklärt, ist die, dals in der Molybdänsäure Sauerstoffatome einfach ersetzt werden durch labile Sauerstoffdoppelatome, wie man durch folgendes Schema ausdrücken könnte:

$$
\underset{\mathrm{O}-\mathrm{H}}{\mathrm{O}-\mathrm{H}}+\mathrm{O}=\mathrm{MoO}_{2}=\stackrel{\mathrm{O}}{\mathrm{O}}>\mathrm{MoO}_{2}+\mathrm{H}_{2} \mathrm{O} \text {. }
$$

Analog verläuft die Einwirkung bei den Salzen, und es ist interessant, dafs, soweit die Beobachtungen gehen, immer die Zusammensetzung der sauerstoffreichen Salze analog ist derjenigen der gewöhnlichen Molybdate. Das $7 / 3$-fach saure Ammonmolybdat ist das beständigste; in den beiden Permolybdaten ist das Verhältnis $\mathrm{NH}_{4}$ : Mo dasselbe geblieben; ähnlich ist es beim Kalium, Verhältnis $1: 3$. Für die Konstitution der drei Ammoniumsalze könnte man 
folgende Schemata sich denken, die das, was wir meinen, deutlich machen:

1. Gewöhnliches Molybdat:

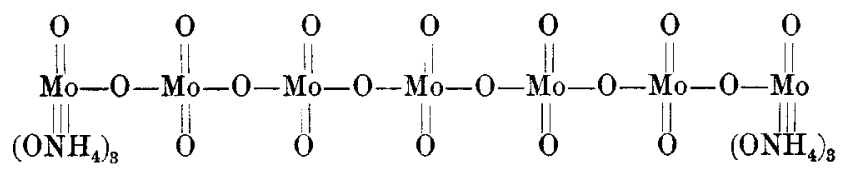

2. Rotes Permolybdat:

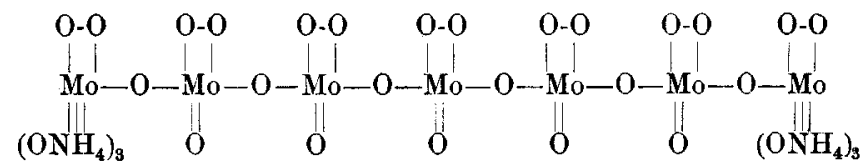

3. Gelbes Permolybdat:

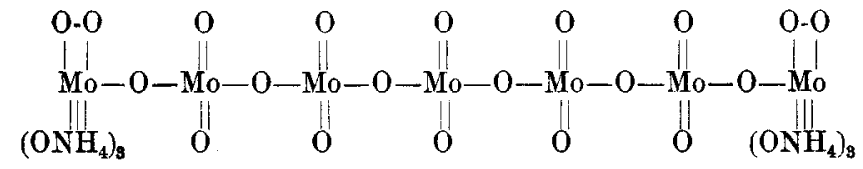

Es erklärt sich mit Hilfe dieser Annahme auch am besten der Wechsel des Verhältnisses zwischen Molybdän und Sauerstoff.

Von sonstigen Reaktionen der beschriebenen Salze seien folgende erwähnt:

Die Temperaturen, bei denen sich die Körper vollkommen zersetzen und entfärben, sind folgende:

$$
\begin{array}{ll}
3\left(\mathrm{NH}_{4}\right)_{2} \mathrm{O} .7 \mathrm{MoO}_{4} \cdot 12 \mathrm{H}_{2} \mathrm{O} . & 105^{\circ} \\
3\left(\mathrm{NH}_{4}\right)_{2} \mathrm{O} .5 \mathrm{MoO}_{3} \cdot 2 \mathrm{MoO}_{4} \cdot 6 \mathrm{H}_{3} \mathrm{O} & 170^{\circ} \\
\mathrm{K}_{2} \mathrm{O} .2 \mathrm{MoO}_{3} \cdot \mathrm{MoO}_{4} \cdot 3 \mathrm{H}_{2} \mathrm{O} \text {. } & 180^{\circ}
\end{array}
$$

Verhalten gegen Natriumphosphat. In verdünnten, mit Salpetersäure angesäuerten Lösungen entsteht keine Fällung, das sauerstoffreichste Ammonsalz giebt auch in konz. saurer Lösung keine Reaktion; das sauerstoffärmere dagegen wohl, und zwar entsteht um so mehr Phosphomolybdat, je konzentrierter die Lösung ist. In zwei Versuchen wurde $31.65 \%$ und $39.91 \%$ der Molybdänsäure im Niederschlag gefunden. Der gesamte Sauerstoff befand sich immer im Filtrat, wie durch Titration mit Permanganat festgestellt werden konnte.

Daraus folgt, dafs, wenn in die Molybdänsäure die O-O-Gruppe für 0 eintritt, die Fähigkeit zur Bildung von komplexen Säuren mit Phosphorsäure verloren geht.

Die Elektrolyse einer wässerigen Lösung des roten Ammonsalzes 
ergab folgendes: An der Anode lebhafte Sauerstoffentwickelung; an der Kathode Entwickelung von Wasserstoff, Reduktion der gelben Lösung unter Entfärbung und schliefslich Abscheidung niederer Oxyde des Molybdäns.

Zum Schlusse möchten wir noch bemerken, dals die Resultate obiger Arbeit in Einklang stehen mit den Ergebnissen einer Untersuchung von A. Prcciñ über Fluooxypermolybdate. ${ }^{1}$ Die Fluooxy. molybdate nämlich nehmen beim Behandeln mit Wasserstoffsuperoxyd ebenfalls unter Gelbfärbung Sauerstoff auf und zwar auf je ein Atom Molybdän ein Atom Sauerstoff. Dies spricht, wie man sieht, für unsere, und gegen Ptchard's Anschauung.

Wir sind zur Zeit damit beschäftigt, die Einwirkung von Wasserstoffsuperoxyd auf andere Molybdate, sowie auf Wolframate zu studieren.

1 Z. anorg. Chem. (1892) 1, 52.

München, Chem. Laboratorium der kgl. Akademie der Wissenschaften.

Bei der Redaktion eingegangen am 21. Februar 1898. 